\title{
Asymptotic decay of the pair correlation function in molecular fluids: Application to hard rods
}

\author{
S. V. Savenko and Marjolein Dijkstra \\ Debye Institute, Soft Condensed Matter Group, Utrecht University, Princetonplein 5, 3584 CC Utrecht, The Netherlands
}

(Received 6 April 2005; published 18 August 2005)

\begin{abstract}
We investigate the asymptotic decay of the total correlation function $h(1,2)$ in molecular fluids. To this end, we expand the angular dependence of $h(1,2)$ and the direct correlation function $c(1,2)$ in the Ornstein-Zernike equation in a complete set of rotational invariants. We show that all the harmonic expansion coefficients $h_{l_{1} l_{2} l}(r)$ are governed by a common exponential decay length and a common wavelength of oscillations in the isotropic phase. We determine the asymptotic decay of the total correlation functions by investigating the pole structure of the reciprocal (q-space) harmonic expansion coefficients $h_{l_{1} l_{2} l}(q)$. The expansion coefficients in laboratory frame of reference $h_{l_{1} l_{2} l}(r)$ are calculated in computer simulations for an isotropic fluid of hard spherocylinders. We find that the asymptotic decay of $h(1,2)$ is exponentially damped oscillatory for hard spherocylinders with a length-to-diameter ratio $L / D \leqslant 10$ for all statepoints in the isotropic fluid phase. We compare our results on the pole structure using different theoretical Ansätze for $c(1,2)$ for hard ellipsoids. The theoretical results show that the asymptotic decay of $h(1,2)$ is exponentially damped oscillatory for all elongations of the ellipsoids.
\end{abstract}

DOI: 10.1103/PhysRevE.72.021202

PACS number(s): 61.20.-p, 61.25.Em, 61.30.- -v

\section{INTRODUCTION}

The asymptotic analysis of pair correlation functions dates back to the work of Kirkwood [1], who showed that the total correlation function $h(r)$ of a fluid with interatomic potentials decaying faster than a power law can be presented as a sum of exponential contributions $r h(r)$ $=\Sigma_{n} \mathcal{A}_{n} e^{-\alpha_{0, n} r} \cos \left(\alpha_{1, n} r+\theta_{n}\right)$ with $\mathcal{A}_{n}$ and $\theta_{n}$ the amplitude and phase, respectively. Consequently, the asymptotics, i.e., the ultimate or leading decay of $h(r)$, is determined by the pole or poles closest to the real axis (smallest $\alpha_{0, n}$ ).

The asymptotic behavior of the pair correlation function plays a significant role in the understanding of interfacial phenomena in fluids, e.g., it has an impact on the wetting transition at wall-fluid interfaces [2,3] as well as on the structure of interfaces between coexisting phases $[2,4,5]$. For high temperatures and densities the asymptotic behavior is determined by repulsive forces, while at low densities and temperatures attractive forces are more important and correlations exhibit monotonic decay. Considering a onedimensional model, Fisher and Widom showed that there exists a line in the temperature-density plane where the asymptotic decay of the pair correlation function crosses over from monotonic to exponentially damped oscillatory behavior [6]. The authors also conjectured that a similar transition should occur in three-dimensional systems provided that the potential is short-ranged. The Fisher-Widom line was calculated for three-dimensional model fluids, e.g., the square-well fluid [3], Lennard-Jones fluid [7,8], (screened) Coulombic fluids $[9,10]$, hard-core attractive Yukawa fluids [11], general binary mixtures [12], screened-Coulomb charged hard-sphere binary fluids [13], binary Gaussian core mixtures [14], colloid-polymer mixtures [15], binary starpolymer solutions [16], and binary hard-sphere mixtures $[17,18]$. The asymptotic decay of the radial distribution function $g(r)$ is determined by investigating the pole structure of the structure factor $S(q)[3,12]$. Moreover, the asymptotic decay was also studied recently in computer simulations for a truncated Lennard-Jones and a hard-sphere fluid [19]. Here, the longest range decay of the total correlation function $h(r)=g(r)-1$ was determined by calculating the direct correlation function $c(r)$ from the simulated $h(r)$ using the Ornstein-Zernike equation. In conclusion, the asymptotic decay of pair correlations in simple fluids is well-studied by now. This should be contrasted to molecular fluids, which show the existence of orientational degrees of freedom that interplay in a nontrivial way with the translational degrees of freedom. To the best of our knowledge, we are not aware of any study on the asymptotic decay of the total correlation function $h(1,2)$ in fluids interacting with anisotropic pair potentials.

In this paper, we give a brief summary of the asymptotics of the pair correlation function of a fluid with short-ranged spherically symmetric pair potentials and we give a generalization for fluids interacting with anisotropic interaction potentials. Expanding the Ornstein-Zernike (OZ) equation in a complete set of rotational invariants, we show that in the isotropic phase the asymptotic decay of all $\mathbf{r}$-frame harmonic expansion coefficients $h_{l_{1} l_{2} l}(r)$ is governed by a common exponential decay length and a common wavelength of oscillations. The asymptotic decay is determined by the pole with the slowest exponential decay of the q-space harmonic expansion coefficient $h_{l_{1} l_{2} l}(q)$, which is related through a Hankel transform to the r-space harmonic expansion coefficient $h_{l_{1} l_{2} l}(r)$. Analogous to our previous study of the truncated Lennard-Jones fluid [19], we measure the space-fixed harmonic expansion coefficients of the total correlation function $h_{l_{1} l_{2} l}(r)$, extract the expansion coefficients of the direct correlation function $c_{l_{1} l_{2} l}(r)$ using the harmonic expansion of the $\mathrm{OZ}$ equation, and determine the asymptotic behavior from the latter. We compare our results for $c(1,2)$ with some theoretical Ansätze for $c(1,2)$.

The paper is organized as follows: in Sec. II, we briefly discuss the asymptotics of the pair correlation function of a 
simple fluid with spherically symmetric pair potentials, and we give a generalization for fluids interacting with anisotropic pair potentials. In Sec. III, we present details on the simulations, from which we obtain the direct correlation function. The results of $c(1,2)$ are compared with several theoretical Ansätze. Finally, the results are discussed in Sec. IV.

\section{THEORY}

\section{A. Simple fluids}

Below, we give a brief outline of the asymptotic decay of the pair correlation function of a fluid with short-ranged, spherically symmetric interaction potentials. For more details, we refer the reader to Refs. [3,12]. The asymptotics of the total pair correlation function $h(r)=g(r)-1$ is most easily determined by investigating the pole structure of the structure factor $S(q)$.

In an isotropic homogeneous bulk fluid, all correlation functions depend on the absolute distance and the OrnsteinZernike (OZ) equation reads

$$
h(r)=c(r)+\rho \int d \mathbf{r}^{\prime} c\left(\left|\mathbf{r}-\mathbf{r}^{\prime}\right|\right) h\left(r^{\prime}\right),
$$

where $\rho$ is the bulk density. The OZ equation relates the total pair correlation function $h(r)$ to the direct correlation function $c(r)$. The three-dimensional Fourier transform $\hat{f}(q)$ of a spherically symmetric function $f(r)$ reads

$$
\begin{aligned}
& \hat{f}(q)=4 \pi \int_{0}^{\infty} d r r^{2} f(r) \frac{\sin q r}{q r}, \\
& f(r)=\frac{1}{2 \pi^{2}} \int_{0}^{\infty} d q q^{2} \hat{f}(q) \frac{\sin q r}{q r},
\end{aligned}
$$

and the OZ equation (1) in Fourier representation reads

$$
\hat{h}(q)=\frac{\hat{c}(q)}{1-\rho \hat{c}(q)},
$$

where the convolution theorem is used. Applying the inverse Fourier transform, one obtains

$$
r h(r)=\frac{1}{4 \pi^{2} i} \int_{-\infty}^{\infty} d q q e^{i q r} \frac{\hat{c}(q)}{1-\rho \hat{c}(q)} .
$$

For short-ranged or exponentially decaying pair potentials, where $c(r)$ decays faster than a power law, the asymptotic behavior of $r h(r)$ is determined by the poles of $\hat{h}(q)$, i.e., complex values of the wave number $q=\alpha_{1}+i \alpha_{0}$ that satisfy

$$
1-\rho \hat{c}(q)=0 .
$$

Here $\alpha_{0}, \alpha_{1} \in \mathbb{R}$. Eq. (4) can be evaluated by performing the contour integration on an infinite semicircle in the upper half plane, and provided all poles are simple, one obtains

$$
r h(r)=\sum_{n} R_{n} e^{i q_{n} r},
$$

where $q_{n}$ is the $n$th pole and $2 \pi R_{n}$ is the corresponding residue of $q \hat{c}(q) /[1-\rho \hat{c}(q)]$ at $q=q_{n}$. The poles can be found by equating the real and imaginary part in Eq. (5), yielding the following set of equations for $\alpha_{1}$ and $\alpha_{0}$ :

$$
\left\{\begin{array}{l}
1=4 \pi \rho \int_{0}^{\infty} d r r^{2} c(r) \frac{\sinh \left(\alpha_{0} r\right)}{\alpha_{0} r} \cos \left(\alpha_{1} r\right) \\
1=4 \pi \rho \int_{0}^{\infty} d r r^{2} c(r) \cosh \left(\alpha_{0} r\right) \frac{\sin \left(\alpha_{1} r\right)}{\alpha_{1} r} .
\end{array}\right.
$$

Provided $c(r)$ is known for a given state point, this set of equations can be used to find the poles. In general, an infinite number of poles can be expected. However, the pole or poles with the smallest imaginary part of $q_{n}$ has the slowest exponential decay, and dominates the asymptotic behavior or longest range part of $h(r)$. Two scenarios are possible: (i) Pure exponential decay dominates at longest range, if a pole lying on the imaginary axis, i.e., $q=i \alpha_{0}$, has the smallest value of $\alpha_{0}$. The pure imaginary pole can be obtained from the first equation of Eq. (7) with $\alpha_{1}=0$. Using Eq. (6), we find that the contribution of a pure imaginary pole to $r h(r)$ is $r h(r) \sim \mathcal{A} \exp \left(-\alpha_{0} r\right)$ with $2 \pi \mathcal{A}$ the corresponding residue. (ii) Exponentially damped oscillatory decay with a wavelength $2 \pi / \alpha_{1}$ dominates at longest range, if a conjugate pair of poles $q= \pm \alpha_{1}+i \alpha_{0}$ has a smaller imaginary part than the pure imaginary pole. The decay of $r h(r)$ at longest range is then determined by the contribution of the conjugate pair of complex poles: $r h(r) \sim 2|\mathcal{A}| \exp \left(-\alpha_{0} r\right) \cos \left(\alpha_{1} r-\theta\right)$. Explicit formulas for the amplitude $|\mathcal{A}|$ and phase $\theta$ are given elsewhere $[10,12]$.

For potentials, which are purely repulsive and of finite range, $c(r)$ is predominately negative for all $r$ and hence no solution can be found for a pure imaginary pole. We mention that the rapidly decaying tail in $c(r)$ outside the core, which was found in simulations [20], does not play a significant role for the asymptotic decay [19]. Consequently, one expects exponentially damped oscillatory decay for fluids interacting with purely repulsive short-ranged potentials.

\section{B. Molecular fluids}

In molecular liquids, the interaction potential and hence the correlation functions, depend no longer only on the absolute center-of-mass distance of the two particles, but also on their orientations. We stress that this even holds in the isotropic phase. Consequently, the structure in molecular fluids is described by the total correlation function $h\left(\mathbf{r}_{1}, \mathbf{u}_{1}, \mathbf{r}_{2}, \mathbf{u}_{2}\right) \equiv h(1,2)$, where $\mathbf{r}_{1}$ and $\mathbf{r}_{2}$ are the center-ofmass coordinates of particle 1 and 2 , and $\mathbf{u}_{1}$ and $\mathbf{u}_{2}$ denote the unit vectors defining the orientations of the molecules. The interaction potential in molecular systems is usually very complicated, i.e., Lennard-Jones potentials for each individual atom in a molecule and thus the orientational degrees of freedom mixes in a nontrivial way with the translational degrees of freedom. In this paper, we limit ourselves to uniaxially symmetric particles, which are invariant under in- 
version of their principle axis. A simple model to study the interplay of translational and rotational degrees of freedom is a fluid consisting of hard spherocylinders or hard ellipsoids of revolution. These systems serve as simple models for molecular fluids and lyotropic liquid crystals.

It is convenient to expand the total correlation function in a complete set of angular functions with expansion coefficients depending on the separation $r=\left|\mathbf{r}_{12}\right|$ with $\mathbf{r}_{12} \equiv \mathbf{r}_{1}-\mathbf{r}_{2}$ [21]. There are two common choices for this expansion. In one expansion, the molecular orientations are referred to an intermolecular reference frame in which the polar axis is along the intermolecular vector $\mathbf{r}_{12}$. In the other expansion, the molecular orientations are defined in a space-fixed or laboratory frame of reference. Here, we employ the latter expansion:

$$
f\left(\mathbf{r}, \mathbf{u}_{1}, \mathbf{u}_{2}\right)=\sum_{l_{1} l_{2} l} f_{l_{1} l_{2} l}(r) \Phi_{l_{1} l_{2} l}\left(\mathbf{u}_{1}, \mathbf{u}_{2}, \mathbf{u}_{r}\right),
$$

where $\mathbf{u}_{r}$ is the unit vector in the direction of $\mathbf{r}_{12}$, and $f_{l_{1} l_{2} l}(r)$ are the harmonic expansion coefficients based on a laboratory reference frame. The rotational invariants $\Phi_{l_{1} l_{2} l}\left(\mathbf{u}_{1}, \mathbf{u}_{2}, \mathbf{u}_{r}\right)$ are given by [22]

$$
\begin{aligned}
& \Phi_{l_{1} l_{2} l}\left(\mathbf{u}_{1}, \mathbf{u}_{2}, \mathbf{u}_{r}\right) \\
& \quad=\sum_{m_{1} m_{2} m} C\left(l_{1} l_{2} l ; m_{1} m_{2} m\right) Y_{l_{1} m_{1}}\left(\mathbf{u}_{1}\right) Y_{l_{2} m_{2}}\left(\mathbf{u}_{2}\right) Y_{l m}^{*}\left(\mathbf{u}_{r}\right)
\end{aligned}
$$

with $C\left(l_{1} l_{2} l ; m_{1} m_{2} m\right)$ the Clebsch-Gordon coefficient, $Y_{l m}(\mathbf{u})$ the spherical harmonics, and $*$ indicates the complex conjugate. Selection rules require that $l_{1}, l_{2}, l$ form a triangle, for example, $l$ must obey $\left|l_{1}-l_{2}\right| \leqslant l \leqslant\left(l_{1}+l_{2}\right)[21]$.

Since our particles have uniaxial and head-to-tail symmetry, $l_{1}, l_{2}, l$ are all even and the harmonic expansion coefficients satisfy the symmetry property $h_{l_{1} l_{2}}(r)=h_{l_{2} l_{1} l}(r)$ [22]. Moreover, all of the harmonic expansion coefficients are real, and consequently, their transforms in Fourier space are even.

The direct correlation function $c(1,2) \equiv c\left(\mathbf{r}_{1}, \mathbf{u}_{1}, \mathbf{r}_{2}, \mathbf{u}_{2}\right)$ is defined through the Ornstein-Zernike equation

$$
h(1,2)=c(1,2)+\frac{\rho}{4 \pi} \int d \mathbf{r}_{3} d \mathbf{u}_{3} c(1,3) h(3,2),
$$

where $\rho$ is the number density. Expanding $c(1,2)$ and $h(1,2)$ in rotational invariants (8), and applying the Fourier transform, one obtains [21]

$$
h_{l_{1} l_{2} l}(q)=c_{l_{1} l_{2} l}(q)+(4 \pi)^{-3 / 2} \rho \sum_{l_{3} l^{\prime} l^{\prime \prime}} h_{l_{3} l_{2} l^{\prime \prime}}(q) c_{l_{1} l_{3} l^{\prime \prime}}(q) K_{l^{\prime \prime} l^{\prime} l_{3}}^{l_{1} l_{2} l_{3}}
$$

with the coefficients

$$
K_{l^{\prime \prime} l^{\prime} l_{3}}^{l_{1} l_{2} l}=\left(2 l^{\prime}+1\right)\left(2 l^{\prime \prime}+1\right)\left(\begin{array}{ccc}
l^{\prime} & l^{\prime \prime} & l \\
0 & 0 & 0
\end{array}\right)\left\{\begin{array}{lll}
l_{1} & l_{2} & l \\
l^{\prime \prime} & l^{\prime} & l_{3}
\end{array}\right\},
$$

where

$$
\left(\begin{array}{lll}
l^{\prime} & l^{\prime \prime} & l \\
0 & 0 & 0
\end{array}\right) \text { and }\left\{\begin{array}{lll}
l_{1} & l_{2} & l \\
l^{\prime \prime} & l^{\prime} & l_{3}
\end{array}\right\}
$$

are the $3 j$ and $6 j$ symbols, respectively. Here the $\mathbf{r}$-space harmonic expansion coefficients $f_{l_{1} l_{2} l}(r)$ are related to the q-space harmonic expansion coefficients $f_{l_{1} l_{2} l}(q)$ through a Hankel transform

$$
\begin{gathered}
f_{l_{1} l_{2} l}(q)=4 \pi i^{l} \int_{0}^{\infty} d r r^{2} j_{l}(q r) f_{l_{1} l_{2} l}(r), \\
f_{l_{1} l_{2} l}(r)=\frac{4 \pi}{(2 \pi)^{3}}(-i)^{l} \int_{0}^{\infty} d q q^{2} j_{l}(q r) f_{l_{1} l_{2} l}(q),
\end{gathered}
$$

where $j_{l}(q r)$ is the spherical Bessel function. For $l=0$, the Hankel transform coincides with the Fourier transform (2).

The pole analysis for simple fluids described in Sec. II A can be extended to molecular fluids. If we truncate the expansion by imposing an upper limit $l_{1}, l_{2}, l \leqslant l_{\max }$, the set (11) can be solved by rewriting it as a simple matrix equation $A_{i j} h_{j}=c_{i}$. The component $j$ of vector $h$ is short for $j=\left(l_{1} l_{2} l\right)$, the $q$ dependence is dropped for clarity and the elements of matrix $A_{i j}$ are functions of $c_{l_{1} l_{2}}(q)$. We give explicit expressions for $A_{i j}$ in Appendix B for $l_{\max }=2$. It is straightforward to derive that $h_{j}=\left(A^{-1}\right)_{j i} c_{i}$ where $\left(A^{-1}\right)_{j i}=|A|_{i j} /|A|$ with $|A|_{i j}$ the cofactor of matrix element $A_{j i}$. Hence $h_{l_{1} l_{2} l}(q)$ can be expressed as a function of $c_{l_{1} l_{2} l}(q)$ in the form $h_{l_{1} l_{2} l}(q)$ $=D_{l_{1} l_{2} l}(q) / D(q)$. Here $D(q)=|A|$ is the determinant of the coefficients matrix of Eq. (11) and the determinants $D_{l_{1} l_{2} l}(q)$ are dependent on the indices $\left(l_{1} l_{2} l\right)$. Both determinants are polynomials in $c_{l_{1} l_{2} l}(q)$ [23].

The total pair correlation function can now be expressed as

$$
h_{l_{1} l_{2} l}(r)=\frac{(-i)^{l}}{2 \pi^{2}} \int_{0}^{\infty} d q q^{2} j_{l}(q r) \frac{D_{l_{1} l_{2} l}(q)}{D(q)} .
$$

By analogy with Eq. (4), the asymptotic behavior of $h_{l_{1} l_{2}}(r)$ is determined by the poles of $h_{l_{1} l_{2} l}(q)$. This is similar to the case of binary mixtures, for which it was shown explicitly that a common denominator exists for all partial pair correlation functions in Ref. [12]. We stress that the situation here is more complicated than in the case of binary mixtures, although formally there exists a common denominator for all $h_{l_{1} l_{2} l}(q)$ [12]. The matrix equation (11) decouples into separate subsets each corresponding to a different value of $l_{2}$. In Appendix A we show explicitly that in the case of binary mixtures the determinant of the subsets are all equal. For molecular fluids, the coefficients matrix of Eq. (11) has a block structure, but the dimension of the determinants varies for different values of $l_{2}$ as the selection rules exclude certain values of $l_{1}, l_{2}, l$ for the expansion coefficients. We now define $\left|A^{\left(l_{2}\right)}\right|$ as the determinant of block $l_{2}$. The expansion coefficient can be expressed as $h_{j}=\left(A^{-1}\right)_{j i} c_{i}$ where $\left(A^{-1}\right)_{j i}=|A|_{i j} /|A| \quad$ with $\quad|A|=\prod_{l_{2}^{\prime}}\left|A^{\left(l_{2}^{\prime}\right)}\right|, \quad$ and $\quad|A|_{i j}$ $\left.=\left|A^{\left(l_{2}\right)}\right|_{i j} \Pi_{l_{2}^{\prime} \neq l_{2}} \mid A^{\left(l_{2}^{\prime}\right)}\right) \mid$. Hence $\left(A^{-1}\right)_{j i}$ reduces to $\left(A^{-1}\right)_{j i}$ $=\left|A^{\left(l_{2}\right)}{ }_{i j} /\right| A^{\left(l_{2}\right)} \mid$. The asymptotic behaviour of the total corre- 
lation functions is determined by the poles and residues of $h_{j}$ and are thus determined by the zeros of the denominator $\left|A^{\left(l_{2}\right)}\right|$.

Consequently, only the harmonics belonging to a particular subset corresponding to a certain value of $l_{2}$ share the same determinant and the same pole structure. It should be noted that the determinants for all the subsets contain all the $c_{l_{1} l_{2} l}(q)$ expansion coefficients, which are allowed by the selection rules.

Employing the symmetry property that the harmonic expansion coefficients are invariant under $l_{1} \leftrightarrow l_{2}$ permutation, we can conclude that determinants corresponding to blocks with different values of $l_{2}$, being not equal in general, do share the zeros, and thus all the expansion coefficients again share the same pole structure.

This is similar to the case of (binary) mixtures, where the total pair correlation functions $h_{\alpha \beta}(r)$ of species $\alpha$ and $\beta$ possess the same characteristic decay length and, if present, oscillatory wavelength for the asymptotic decay $[12,14]$. This analogy is clear as each harmonic expansion coefficient in the expansion of the Ornstein-Zernike equation can be regarded as a separate species in a multi component mixture. It also means that the decay of correlations between two molecules at large distances from each other does not depend on their relative orientations but is determined by the integral properties of the fluid in between. We note that the amplitudes $A$ and the phases $\theta$ are dependent on the indices $\left(l_{1} l_{2} l\right)$.

As mentioned before, Eq. (11) separates into independent subsets corresponding to different values of $l_{2}$. The most convenient subset is certainly the one corresponding to $l_{2}$ $=0$. Selection rules imply that the only nontrivial expansion coefficients in this set are those of type $h_{l 0 l}(q) \equiv h_{l}(q)$. The $6 j$ symbol contains now a zero in one of the six positions. Employing the symmetry property that the $6 j$ symbol is invariant under permutations of the columns, one can evaluate the $6 j$ symbol as follows:

$$
\begin{aligned}
\left\{\begin{array}{lll}
l_{1} & 0 & l \\
l^{\prime \prime} & l^{\prime} & l_{3}
\end{array}\right\} & =\left\{\begin{array}{lll}
l_{1} & l & 0 \\
l^{\prime \prime} & l_{3} & l^{\prime}
\end{array}\right\} \\
& =(-)^{l_{1}+l^{\prime \prime}+l^{\prime}}\left[\left(2 l_{1}+1\right)\left(2 l^{\prime \prime}+1\right)\right]^{-1 / 2} \delta_{l_{1} l} \delta_{l^{\prime \prime} l_{3}} .
\end{aligned}
$$

The corresponding set simplifies to a simple matrix equation, which reads

$$
h_{l}(q)=c_{l}(q)+\rho \sum_{k} b_{l k}(q) h_{k}(q)
$$

where the coefficients $b_{l k}(q)$ are defined as

$$
b_{l k}(q)=(4 \pi)^{-3 / 2} \sqrt{\frac{2 k+1}{2 l+1}} \sum_{m}(2 m+1)\left(\begin{array}{ccc}
m & k & l \\
0 & 0 & 0
\end{array}\right) c_{l k m}(q)
$$

with $b_{l k}(q)=b_{k l}(q)(2 k+1) /(2 l+1)$. The asymptotic behavior of $h_{l 0 l}(r) \equiv h_{l}(r)$ is determined by the poles of $h_{l}(q)$,

$$
D\left[\delta_{l k}-\rho b_{l k}\left(\alpha_{1}+i \alpha_{0}\right)\right]=0 .
$$

Thus the complex values of $q=\alpha_{1}+i \alpha_{0}$, at which one of the eigenvalues of $\rho b_{l k}(q)$ equals 1 , govern not only the asymptotic decay of the harmonic expansion coefficients of type $h_{l 0 l}(r)$ but also of all the other expansion coefficients. The q-space coefficients $b_{l k}(q)$ can be obtained by employing the Hankel transform (13) of $c_{l_{1} l_{2} l}(r)$. In the case of simple fluids, $l_{\max }$ can be set to 0 and Eq. (18) simplifies to Eq. (5). We follow the convention, where $c_{000}(r)$ $=(4 \pi)^{3 / 2} c(r)$, with $c(r)$ the direct correlation function averaged over all possible orientations.

We employ the following expression for the spherical bessel functions:

$$
j_{n}(x)=m_{n+1}(x) \sin (x)+p_{n}(x) \cos (x),
$$

where $m_{n+1}(x)$ and $p_{n}(x)$ are polynomials of order $-(n+1)$ and $-n$, respectively, which can be obtained from the recurrence relations [24]. The integration limits in Eq. (14) can be extended to $(-\infty, \infty)$ and the resulting integral $(20)$ can be carried out by contour integration,

$$
\begin{aligned}
h_{l}(r)= & \frac{(-1)^{l / 2}}{4 \pi^{2}} \int_{-\infty}^{\infty} d q q^{2} \exp (i q r)\left[-i m_{l+1}(q r)+p_{l}(q r)\right] \\
& \times \frac{D_{l}\left[\delta_{l k}-\rho b_{l k}(q)\right]}{D\left[\delta_{l k}-\rho b_{l k}(q)\right]} .
\end{aligned}
$$

Provided all the poles are simple and leaving only the leading order, we find

$$
r^{l+1} h_{l}(r)=\frac{(-)^{l / 2} M_{l+1}}{2 \pi} \sum_{n} q_{n}^{1-l} \frac{D_{l}\left[\delta_{l k}-\rho b_{l k}\left(q_{n}\right)\right]}{D^{\prime}\left[\delta_{l k}-\rho b_{l k}\left(q_{n}\right)\right]} \exp \left(i q_{n} r\right),
$$

where $M_{l+1}$ is the coefficient of the leading power in $m_{l+1}\left(q_{n} r\right)$ and $D^{\prime}\left[\delta_{l k}-\rho b_{l k}\left(q_{n}\right)\right]$ denotes the derivative of the determinant of Eq. (18) at $q=q_{n}$. Equation (21) generalizes the corresponding expression for simple fluids, and can be used to determine the character of asymptotic decay of correlations in molecular fluids provided the harmonic expansion coefficients $c_{l_{1} l_{2} l}(r)$ are known. If the pole with the lowest imaginary value lies on the imaginary axis then the asymptotic decay is pure exponential, otherwise it is determined by a conjugate pair of poles and the asymptotic decay is exponentially damped oscillatory.

The number of harmonic expansion coefficients grows very quickly as $l_{\max }$ increases. However, the determinant in Eq. (18) can be expanded in the density. If we keep only the linear terms in the density, Eq. (18) simplifies to

$$
(4 \pi)^{-3 / 2} \rho \sum_{l m}(2 m+1)\left(\begin{array}{ccc}
m & l & l \\
0 & 0 & 0
\end{array}\right) c_{l l m}\left(\alpha_{1}+i \alpha_{0}\right)=1 .
$$

In the low density approximation only the diagonal expansion coefficients of the form $c_{l l m}(r)$ determine the asymptotic decay of the correlations. 


\section{HARD-ROD FLUIDS}

The occurrence of both pure imaginary and complex poles in simple fluids is a consequence of the fact that the pair potential contains attractive and repulsive parts. In the case of simple fluids interacting with purely repulsive shortranged potentials, the asymptotic decay is exponentially damped oscillatory, as the direct correlation function is negative (or close to zero) for all distances [19]. For instance, the asymptotic decay of $h(r)$ in the hard-sphere fluid is exponentially damped oscillatory for all statepoints, even for very low densities. More significantly, perhaps, we did not find any pure imaginary pole that can give rise to monotonic asymptotic decay, using simulation data [20] for the $c(r)$ of hard spheres [19]. For molecular fluids interacting with purely repulsive short-ranged potentials, there is no such simple argument about whether the asymptotic decay is exponentially damped oscillatory or monotonic. The aim of this paper is to study the asymptotic decay of the pair correlations in fluids consisting of hard spherocylinders and hard ellipsoids. In particular, we explore whether a pure imaginary pole can be present in hard-rod fluids and, if so, we investigate whether there is a crossover from damped oscillatory decay for hard spheres to monotonic decay upon increasing the aspect ratio. To this end, we determine the harmonic expansion coefficients of the direct correlation function $c_{l_{1} l_{2} l}(r)$ by simulations for hard spherocylinders and by different theoretical Ansätze for hard ellipsoids. We describe the simulation results in Sec. III A and the theoretical results in Sec. III B.

\section{A. Simulations}

We perform Monte Carlo simulations of an isotropic fluid of hard spherocylinders, consisting of a cylindrical part with diameter $D$ and length $L$, and capped with hemispheres of diameter $D$ at both ends. Note that the full length of the rod is equal to $L+D$. We wish to determine the harmonic expansion coefficients of the direct correlation function in laboratory frame. The approach is similar to Ref. [19], where the Ornstein-Zernike equation was used to determine the direct correlation function $c(r)$ from the total correlation function $h(r)$ measured directly in simulations. In contrast to experiments, where only information of a few harmonics are directly accessible, computer simulations provide a way to measure all the expansion coefficients of the pair correlation function. To this end, we calculate the harmonic expansion coefficients $g_{l_{1} l_{2} l}(r)$ in bins of width $\Delta r$. By applying the orthogonality condition of the spherical harmonics, Eq. (8) can be inverted to yield [25]

$$
\begin{aligned}
g_{l_{1} l_{2} l}\left(r_{j}\right)= & \frac{(4 \pi)^{3 / 2}\left[\left(2 l_{1}+1\right)\left(2 l_{2}+1\right)(2 l+1)\right]^{1 / 2}}{\rho V\left(r_{j}\right) C\left(l_{1} l_{2} l ; 000\right) N} \\
& \times\left\langle\sum_{i=1}^{n_{j}} P_{l_{1}}\left(\cos \theta_{1}\right) P_{l_{2}}\left(\cos \theta_{2}\right) P_{l}\left(\cos \theta_{r}\right)\right\rangle,
\end{aligned}
$$

where $N$ is the number of particles, $n_{j}$ is the number of par- ticle pairs whose center-of-mass distances are within a range of $r_{j} \pm \Delta r / 2, V\left(r_{j}\right)$ denotes the volume of the spherical shell of thickness $\Delta r$ centered at $r_{j}$, and $C\left(l_{1} l_{2} l ; 000\right)$ are the Clebsch-Gordan coefficients. The standard polar and azimuthal angles $\mathbf{u}_{i}=\left(\theta_{i}, \phi_{i}\right)$ are used for the orientations of particle $i$ with $0 \leqslant \theta_{i} \leqslant \pi$ and $0 \leqslant \phi_{i} \leqslant 2 \pi$.

The only relevant parameters that determine the thermodynamic properties of a fluid of hard spherocylinders are the number density and the length-to-diameter ratio $L / D$. We perform simulations of a system consisting of hard spherocylinders with a length-to-diameter ratio of $L / D=5$ and 10 at densities below the bulk isotropic-nematic transition. The densities of the coexisting isotropic phase read $\eta_{L / D=5}^{I}=0.40$ and $\eta_{L / D=10}^{I} \approx 0.24$ [26]. The simulations are started from an initial configuration with all the center-of-masses of the rods distributed randomly in the simulation box, but all the orientations aligned along one direction. We check for equilibrium by monitoring the nematic order parameter. When equilibrium is reached, we perform production runs of $10^{6}$ sweeps. The harmonic expansion coefficients of the pair correlation function are sampled every sweep up to $l_{\max }=10$. The $g_{l_{1} l_{2} l}(r)$ profiles are accumulated in bins of width $\Delta r=D / 10$. The simulations are computationally more expensive with increasing $L / D$. For instance, the simulation for $L / D=10$ at a density just below the bulk isotropic-nematic transition takes about two months of "500-MHz R14000" processor time. We are therefore restricted to length-to-diameter ratios $L / D$ $\leqslant 10$.

In contrast to earlier simulations, where the expansion coefficients were sampled in molecular frame for calculating $c(1,2)$ in fluids of hard ellipsoids [27], we are able to apply the Hankel transform directly to obtain the set of $g_{l_{1} l_{2} l}(q)$ from which we can extract $h_{l_{1} l_{2} l}(q)$ through $h_{l_{1} l_{2} l}(q)$ $=g_{l_{1} l_{2} l}(q)-(4 \pi)^{3 / 2} \delta_{0 l_{1}} \delta_{0 l_{2}} \delta_{0 l}$. Subsequently, we solve the set (11) to find $c_{l_{1} l_{2} l}(q)$. Applying the inverse Hankel transform, we obtain the harmonic expansion coefficients $c_{l_{1} l_{2} l}(r)$. Figure 1 shows simulation results for a fluid of $N=600-1200$ hard spherocylinders in a volume $V$ with $L / D=10$ and packing fraction $\eta=\left(\pi D^{3} / 6+\pi L D^{2} / 4\right) N / V=0.1592$. Figure 1 shows only a selection of the harmonic expansion coefficients $c_{l_{1} l_{2} l}(r)$. As also found in previous simulation studies $[27,28]$, all the functions are short-ranged. Moreover, the functions decay rapidly outside the overlap region $r>L+D$. At small $r$, the accuracy of $c_{l_{1} l_{2} l}(r)$ decreases significantly with increasing values of $l_{1}, l_{2}, l$, which is due to the truncation of the Hankel transform. However, this does not have a strong impact on the values obtained for the poles [19]. Figure 1 shows that the position-position correlation function $c_{000}(r)$ is negative inside the core, while the orientationorientation correlation function $c_{220}(r)$ is mostly positive, indicating an effective attraction between the rods with similar orientations. Figure 2 shows $c_{000}(r)$ for a hard spherocylinder fluid with $L / D=5$ and 10 at varying packing fractions. The direct correlation function averaged over all possible orientations, i.e., $c_{000}(r)$, is negative inside the core independent of $L / D$, while it oscillates close to zero outside the core. This is similar to the case of hard spheres with diameter $D$. Groot et al. found a rapidly decaying tail in $c(r)$ with $|c(r)| \leqslant 0.01$ 

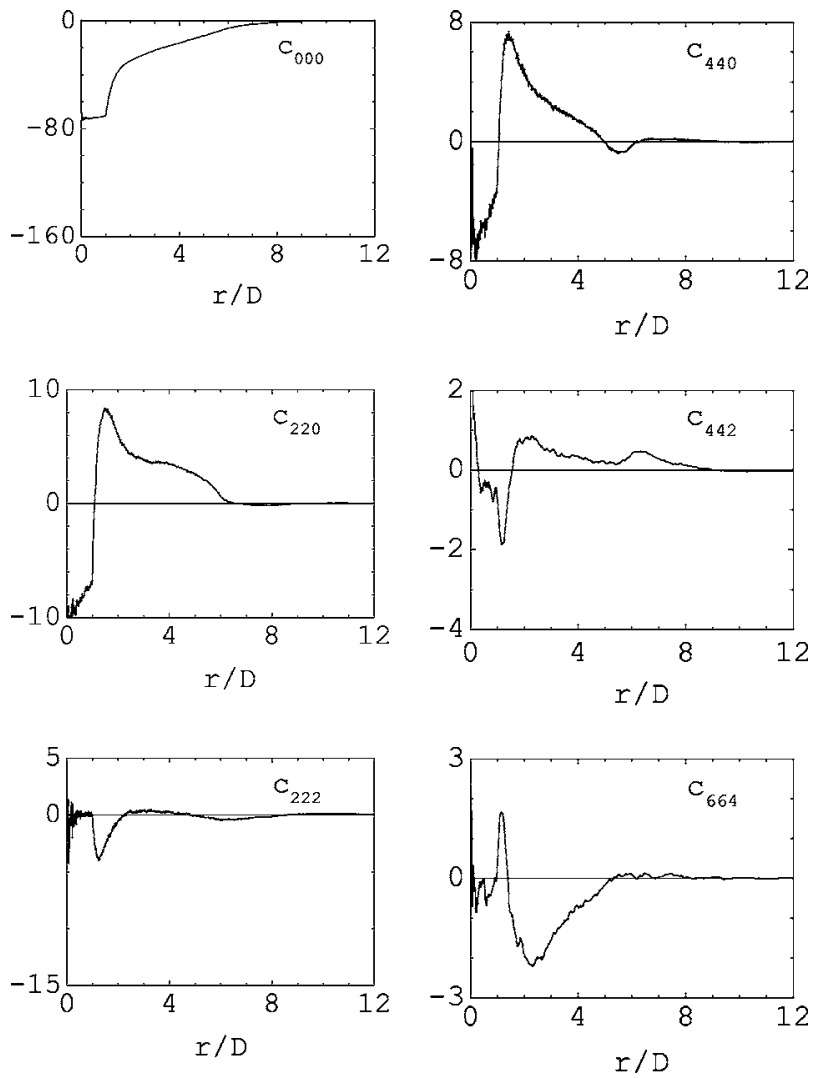

FIG. 1. The selected harmonic expansion coefficients of the direct correlation function $c_{l_{1} l_{2} l}(r)$ with $l_{1} l_{2} l=000,220,222,440,442$, and 664 for a fluid of hard spherocylinders with $L / D=10$ and packing fraction $\eta=0.1592$ obtained from simulations.

for $r>2 D$ using simulation data for $h(r)$ in the $\mathrm{OZ}$ equation [20]. We observe clearly in Fig. 2 that $c_{000}(r)$ becomes more negative inside the core as the packing fraction increases and the effect of repulsion becomes more important.

\section{B. Model functions}

In addition to the simulations, we also use two theoretical model expressions for the direct correlation function. The first one is the Pynn [29] and Wulf [30] Ansatz: $c_{P y n n}(1,2)$ $=c_{H S}(r / \sigma(1,2), \eta)$, where $c_{H S}$ is the hard-sphere function evaluated at the same packing fraction $\eta$ as the molecular fluid, and $\sigma(1,2)=\sigma\left(\hat{u}_{1}, \hat{u}_{2}, \hat{r}_{12}\right)$ is the closest approach distance for given orientations of molecules and center-center vector. The latter can easily be calculated for hard ellipsoids of revolution [31]. Another Ansatz, we employ is due to Parsons [32] and Lee [33]. It approximates the direct correlation function by a density-scaled Mayer function $c_{\text {Parsons }}(1,2)$ $=\varphi(\eta) f(1,2)$. The Mayer function $f(1,2)=-1$ for $r$ $\leqslant \sigma(1,2)$ and $f(1,2)=0$ for $r>\sigma(1,2)$. The weight function reads $\varphi(\eta)=(1-\eta / 4) /(1-\eta)^{4}$, which produces an accurate equation of state for hard spheres. We calculate the harmonic expansion coefficients for $c_{l_{1} l_{2} l}(r)$ of a fluid consisting of hard ellipsoids using the Pynn-Wulf and Parsons-Lee approximations. A selection of $c_{l_{1} l_{2} l}(r)$ is shown in Fig. 3 for hard ellipsoids with an elongation of $a / b=11$ and packing
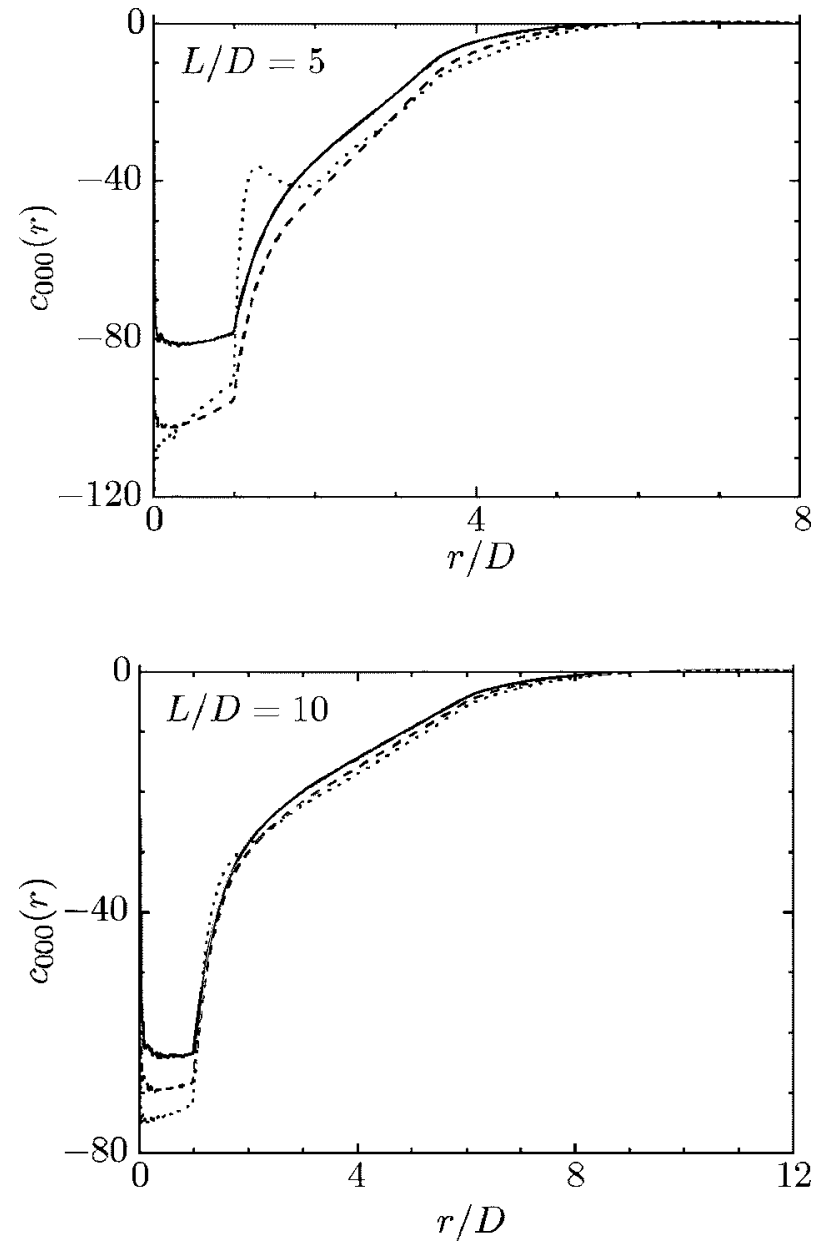

FIG. 2. Simulation results for the harmonic expansion coefficient of the direct correlation function $c_{000}(r)$ for a fluid of hard spherocylinders with a length-to-diameter ratio $L / D=5$ (top) and packing fractions $-\eta=0.1288$, -- $\eta=0.2003, \cdots \eta=0.3694$, and $L / D=10$ (bottom) and packing fractions - $\eta=0.0922,--\eta$ $=0.1257, \cdots \eta=0.1927$.

fraction $\eta=\pi a b^{2} N / 6 V=0.1592$. The major and minor axis of the ellipsoids are denoted by $a$ and $b$, respectively. For the theoretical Ansätze, we calculate only those harmonics that are required for the low density approximation (22) with $l_{\max }=10$. While the particle shape is different in our simulations and in our theories, the overall features of $c_{l_{1} l_{2} l}(r)$ are similar. For instance, in both simulation and theory, $c_{000}(r)$ is negative, and $c_{220}(r)$ and $c_{440}(r)$ are mostly positive inside the overlap region $r / b \leqslant 11$, and decay rapidly outside the overlap region. In contrast to the reasonable agreement of the theoretical Ansätze in the partial overlap region $1 \leqslant r / b$ $\leqslant 11$, they fail dramatically inside the core region $r / b<1$. The Parsons-Lee scaling breaks down inside the core $r / b$ $<1$ as it depends on the Mayer $f(1,2)$ function which is identically equal to -1 . Hence only $c_{000}(r)$ is different from 0 . The Pynn-Wulff approximation predicts an isotropic function in the limit $r \rightarrow 0$ and it fails to describe the harmonics which are not equal to zero at $r=0$. We refer the reader to Ref. [27] for a more detailed comparison of simulation and theory results for $c(1,2)$. 

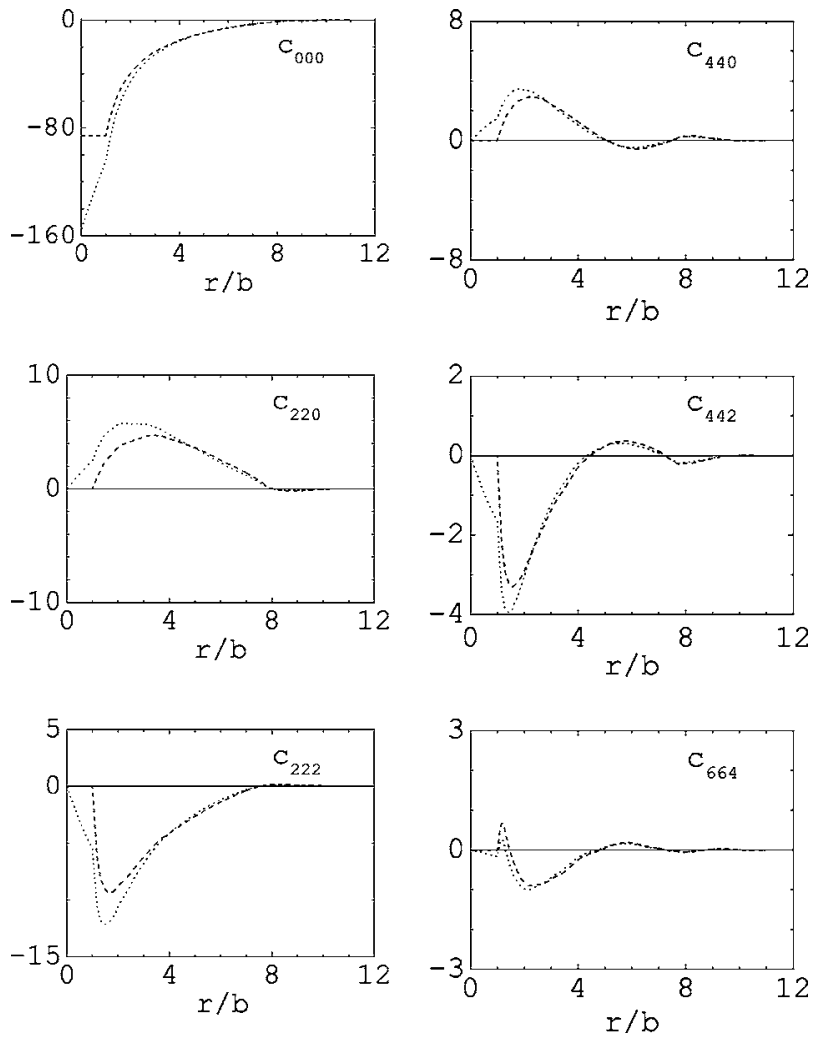

FIG. 3. The selected harmonic expansion coefficients of the direct correlation function $c_{l_{1} l_{2} l}(r)$ with $l_{1} l_{2} l=000,220,222,440,442$, and 664 for a fluid of hard ellipsoids with elongation $a / b=11$ and packing fraction $\eta=0.1592$ obtained from the Pynn-Wulff $[29,30]$ (dotted) and Parsons-Lee [32,33] (short-dashed) Ansätze.

\section{RESULTS AND DISCUSSION}

We investigate the asymptotic decay of the total pair correlation functions for a fluid of hard rods. We obtain the leading poles of $h_{l_{1} l_{2} l}(q)$ for a hard spherocylinder fluid by solving Eq. (18) employing the harmonic expansion coefficients of the direct correlation functions obtained from simulations, which were described in Sec. III A. In order to locate the pure imaginary pole, we consider only the real part of Eq. (18) with the fixed value of $\alpha_{1}=0$. Results for the pure imaginary pole and the pole off the imaginary axis with the smallest value of $\alpha_{0}$ are shown in Fig. 4 for hard spherocylinders with $L / D=5$ and 10 , at varying densities in the isotropic phase ranging from very dilute to just below the density at the bulk isotropic-nematic transition, and $l_{\max }=10$. For comparison, we also plot the results for the leading poles by solving Eq. (7), i.e., using only $c_{000}(r)$, corresponding to $l_{\max }=0$, as in the case of spherically symmetric pair potential, and by solving Eq. (22) with $l_{\max }=10$, which is based on a low density approximation. We also check for convergence with respect to $l_{\max }$ in Eq. (18) for $L / D=10$. The results for $l_{\text {max }}=2$ for the lowest-lying conjugate pair of poles are shown by a dashed line in Fig. 4. We find that it approaches the results of $l_{\max }=10$ very closely. For the pure imaginary pole, we find bad convergence for $l_{\max }=2$, while good agreement is found for $l_{\max }=4$. The crossed squares denote the results for $l_{\max }=4$. For all our simulations, we find that $l_{\max }=10$ is
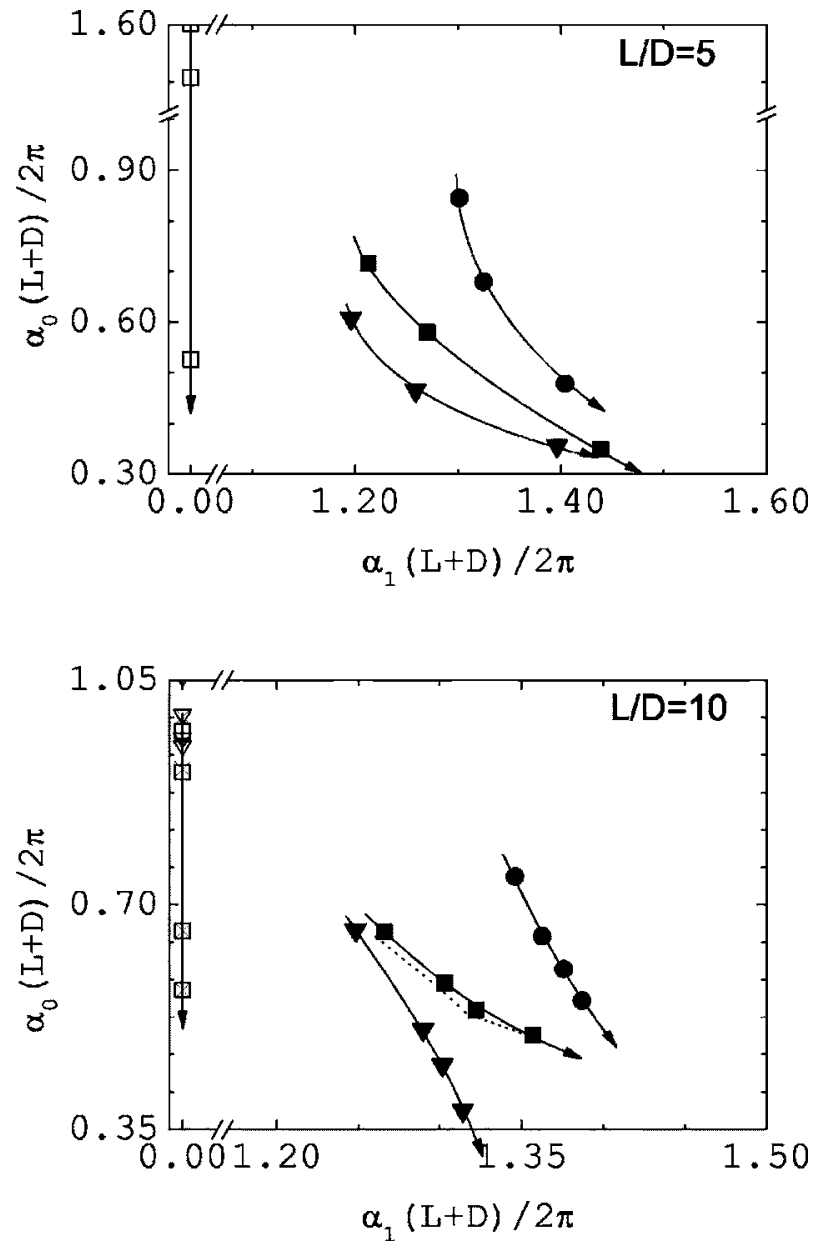

FIG. 4. The imaginary $\left(\alpha_{0}\right)$ and real $\left(\alpha_{1}\right)$ part of the leading poles obtained from simulations of a fluid of hard spherocylinders with a length-to-diameter ratio $L / D=5$ (left) at packing fraction $\eta$ $=0.1288,0.2003$, and 0.3694, and $L / D=10$ (bottom) at $\eta=0.0922$, $0.1257,0.1591$, and 0.1927 . The arrows denote the direction, in which $\eta$ is increased. The symbols with $\alpha_{1}=0$ refer to the pure imaginary pole (open symbols) while those with $\alpha_{1} L / 2 \pi \sim 1.3$ refer to the lowest-lying complex pair $q= \pm \alpha_{1}+i \alpha_{0}$ (filled symbols, only the pole with $\alpha_{1}>0$ is shown). The circles denote the results using the $l_{\text {max }}=0$ approximation (7), the triangles show the results using the low density approximation (22) with $l_{\max }=10$, and the squares the results for $l_{\max }=10$ in Eq. (18). The dashed line show the results for $l_{\max }=2$ in Eq. (18) for the complex pole, while the crossed squares denote the results for $l_{\max }=4$ for the pure imaginary pole, showing the good convergence of $l_{\max }$ for $L / D=10$.

sufficient to provide good convergence. Figure 4 shows that the period $2 \pi / \alpha_{1}$ obtained from the low density approximation (22) is larger than that obtained from the $l_{\text {max }}=0$ approximation (7). On the other hand, the inverse decay length $\alpha_{0}$ from the low density approximation with $l_{\max }=10$ (22) is smaller than that of the $l_{\max }=0$ approximation (7). The poles calculated using $l_{\max }=10$ in Eq. (18) lie between the two approximations of Eqs. (7) and (22).

In the case of the low density approximation (22) and the $l_{\max }=0$ approximation (7), we did not find a pure imaginary pole for $L / D=5$ and we plot only the lowest-lying conjugate pair of poles in Fig. 4 for the two approximations. For 
TABLE I. The occurrence of a pure imaginary pole in simulations of hard spheres $(L / D=0)$ and hard spherocylinders $(L / D=5$ and 10) using different approximations.

\begin{tabular}{cccc}
\hline \hline & \multicolumn{3}{l}{$l_{\max }=10}$, \\
& $\begin{array}{c}l_{\max }=0, \\
\text { Eq. (7) }\end{array}$ & low density & $\begin{array}{c}l_{\max }=10, \\
\text { Eq. (18) }\end{array}$ \\
\hline$L / D=0$ & no & & \\
$L / D=5$ & no & no & yes \\
$L / D=10$ & no & yes & yes \\
\hline \hline
\end{tabular}

$L / D=10$, we do find a pure imaginary pole for the low density approximation (22) using $l_{\max }=10$, while it is absent for the $l_{\max }=0$ approximation (7). In Table I, we summarize the presence or absence of the pure imaginary poles using different approximations and varying $L / D$. We conclude that the appearance of a pure imaginary pole is more related to the positive parts of the higher harmonic expansion coefficients in Eq. (18) than to the tiny positive tail in $c_{000}(r)$ outside the core region. As for $L / D=10$, the pure imaginary pole can even be found using the low density approximation with $l_{\max }=10$ in contrast with $L / D=5$, it is tempting to argue that the occurrence of the monotonic pole is less sensitive for larger $L / D$. In addition, we found good convergence for the value of the monotonic pole using $l_{\max }=4$ in Eq. (18) for $L / D=10$, and we can argue that the occurrence of the monotonic pole is due to the positive part of $c_{220}(r)$ and $c_{440}(r)$.

As expected, the inverse decay length $\alpha_{0}$ decreases with increasing density. The variation of the period $2 \pi / \alpha_{1}$ is weak and changes roughly from $0.85 L$ to $0.70 L$ with increasing density. We find that the imaginary part of both the pure imaginary pole and the lowest-lying complex pole becomes smaller, upon increasing the density toward the value at the isotropic-nematic transition. In addition, the difference between the two imaginary parts decreases with increasing density. However, we find that for all statepoints in Fig. 4, the complex pole has always the smallest value of $\alpha_{0}$. Consequently, the asymptotic decay of the total pair correlation function is exponentially damped oscillatory for a fluid of hard spherocylinders with $L / D \leqslant 10$ at all densities in the isotropic fluid phase. For packing fractions just below the bulk isotropic-nematic transition, the pure imaginary pole and the lowest-lying complex pole $\left[\alpha_{1}(L+D) / 2 \pi, \alpha_{0}(L\right.$ $+D) / 2 \pi]$ are given by $[0,0.53]$ and $[1.44,0.35]$ for $L / D$ $=5$, and for $L / D=10$ by $[0,0.57]$ and $[1.36,0.50]$. The difference between the imaginary parts of the imaginary and the complex pole decreases from 0.18 for $L / D=5$ to 0.07 for $L / D=10$. As our simulations are limited to $L / D \leqslant 10$, we are unable to investigate whether the monotonic pole will have a smaller imaginary part than the complex pole and will become dominant upon increasing the aspect ratio. It remains therefore an open question whether the asymptotic decay of the total pair correlation function crosses over from exponentially damped oscillatory to monotonic upon increasing the aspect ratio.

In addition, we performed very long simulations (5 $\times 10^{6}$ cycles) in order to study the similarity of the decay of the harmonic expansion coefficients. In Fig. 5, we compare

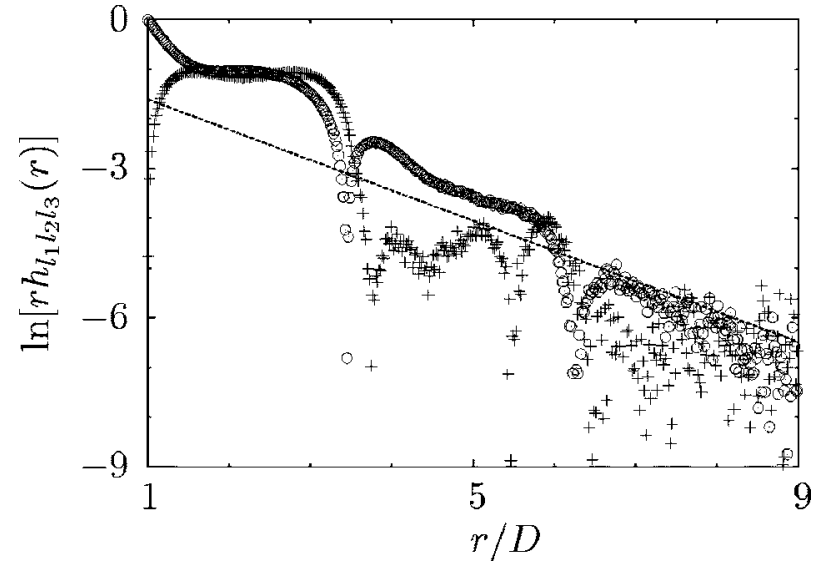

FIG. 5. The harmonic expansion coefficients of the total correlation functions obtained from simulations for a fluid of hard spherocylinders with a length-to-diameter ratio $L / D=5$, and packing fraction $\eta=0.2003: \bigcirc \bigcirc \bigcirc \ln \left[r h_{000}(r)\right],+++\ln \left[r h_{220}(r)\right]$. The dashed line is the slope predicted for the exponential decay using the pole analysis (18).

the simulation results for two harmonic expansion coefficients of the total correlation function $h_{000}(r)$ and $h_{220}(r)$ for $L / D=10$ and $\eta=0.2003$. For comparison, we also show the slope predicted for the exponential decay using the pole analysis (18). As the statistical accuracy of $h_{000}(r)$ and $h_{220}(r)$ is poor, it is hard to make any definite statements on the decay of the harmonics. However, it is tempting to conclude that the two expansion coefficients $h_{000}(r)$ and $h_{220}(r)$ decay similarly at intermediate range and seem to agree with the decay predicted by the pole analysis.

We also employ the theoretical results for the direct correlation function as described in Sec. III B in our pole analysis. We obtain the leading poles of $h_{l_{1} l_{2} l}(q)$ for a fluid of hard ellipsoids using the $l_{\max }=0$ approximation (7) and the low density approximation (22) with $l_{\max }=10$ employing $c_{l_{1} l_{2} l}(r)$ obtained from the Pynn-Wulff and Parsons-Lee Ansätze. We did not find a pure imaginary pole using these Ansätze. Comparing $c_{220}(r)$ and $c_{440}(r)$ in Figs. 1 and 3, we observe that the positive part, which is responsible for the occurrence of the monotonic pole in the simulations, is smaller in the theoretical Ansätze and apparently too weak to predict a pure imaginary pole. Figure 6 shows only results for the lowestlying complex pole with the smallest value of $\alpha_{0}$ for a fluid of hard ellipsoids with an elongation of $a / b=6$ and 11 , at varying densities. The inverse decay length $\alpha_{0}$ decreases upon increasing the density using the Parsons-Lee approximation in agreement with simulations. However, the PynnWulff approximation shows that the value of $\alpha_{0}$ decreases and increases later upon increasing the density.

In Fig. 7, we show the lowest-lying complex pole using the Parsons-Lee approximation for a fluid of hard ellipsoids for several packing fractions and varying elongations 1 $\leqslant a / b \leqslant 25$. We only show results using the low-density approximation (22) with $l_{\max }=10$. Figure 7 shows that the period of the oscillations $2 \pi / \alpha_{1}$ scales roughly with the major axis of the ellipsoids, but decreases slightly upon increasing $a / b$. For an elongation equal to 1 , the results are in agreement with those for hard spheres [19]. 

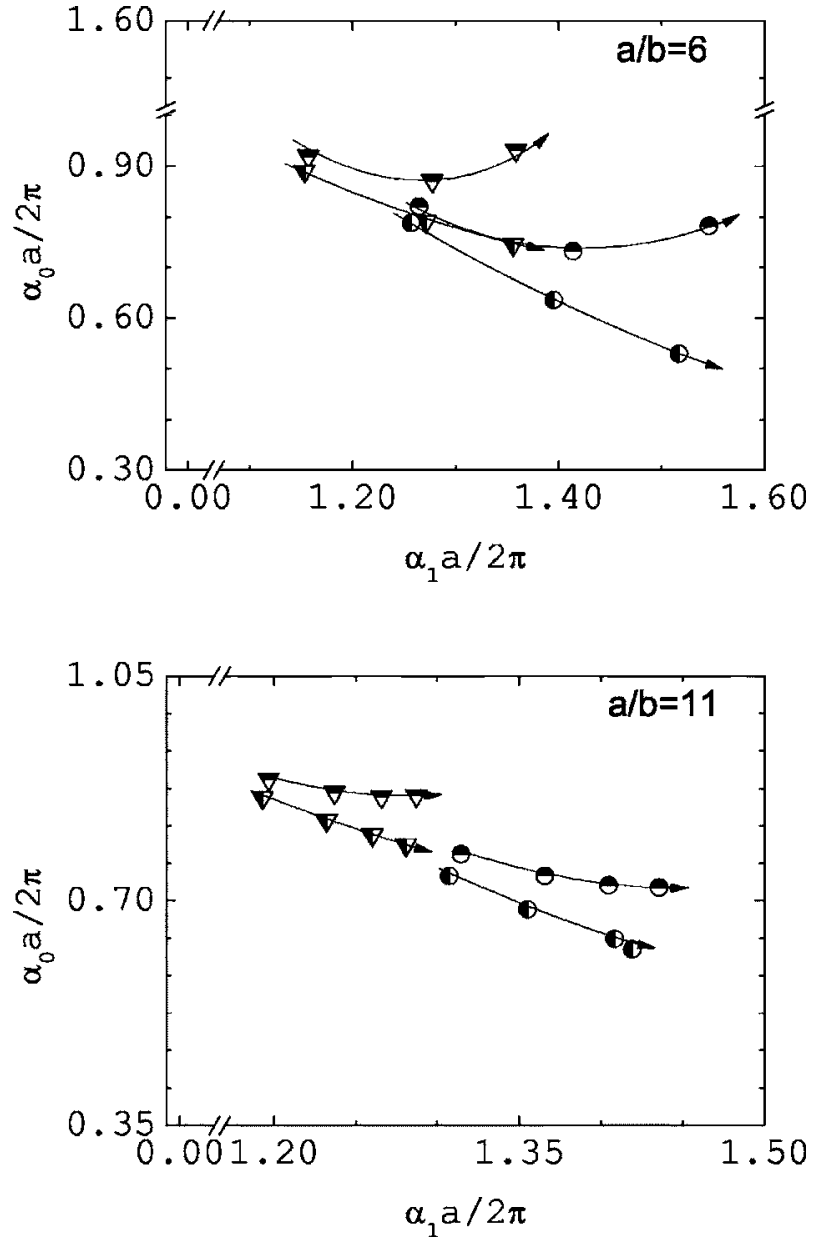

FIG. 6. The imaginary $\left(\alpha_{0}\right)$ and real $\left(\alpha_{1}\right)$ part of the lowestlying conjugate pole $q= \pm \alpha_{1}+i \alpha_{0}$ (only the pole with $\alpha_{1}>0$ is shown) of a fluid of hard ellipsoids with an aspect ratio $a / b=6$ (top) and $a / b=11$ (bottom). The circles denote the results using the $l_{\max }=0$ approximation (7) and the triangles the results using the low-density approximation (22) with $l_{\max }=10$ employing the PynnWulff $[29,30]$ (upper half filled) and the Parsons-Lee [32,33] (left half filled) Ansätze. The arrows denote the direction, in which the density is increased. No pure imaginary pole is found.

In conclusion, we have investigated the asymptotic decay of the total pair correlation functions in fluids consisting of hard spherocylinders and hard ellipsoids. We determined the harmonic expansion coefficients of the direct correlation function $c_{l_{1} l_{2} l}(r)$ by simulations for hard spherocylinders using the $\mathrm{OZ}$ equation and by different theoretical Ansätze for hard ellipsoids. We have shown that the anisotropy of the particles plays an important role in the asymptotic decay of molecular fluids. Although the pair potential is purely repulsive for hard rods, we do find the occurence of a pure imaginary pole in our simulation results. However, for all densities in the isotropic fluid phase, the oscillatory pole has a smaller imaginary part than the pure imaginary pole, and the asymptotic decay of the total pair correlation function is damped oscillatory for length-to-diameter ratios $L / D \leqslant 10$. It remains an open question whether the decay of the total correlation function at longest range crosses over from damped oscillatory to monotonic upon increasing $L / D$. Employing

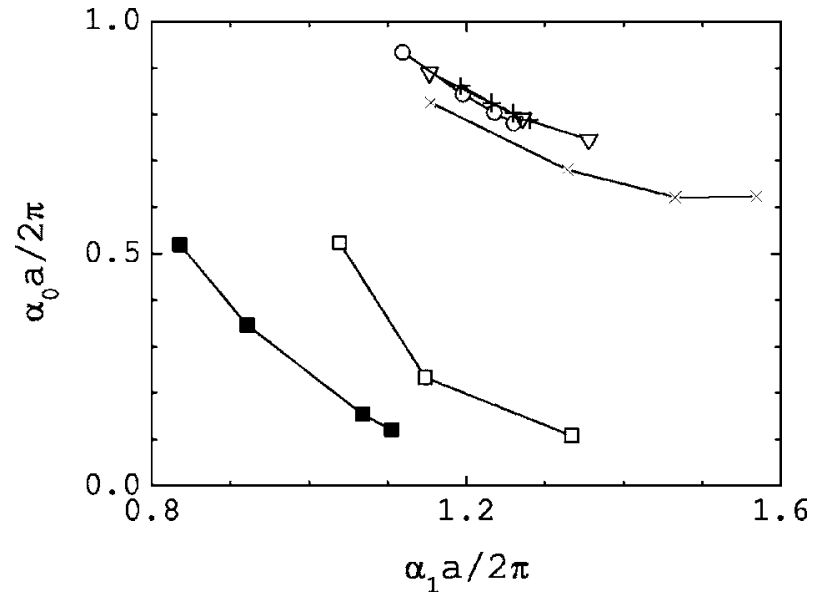

FIG. 7. The imaginary $\left(\alpha_{0}\right)$ and real $\left(\alpha_{1}\right)$ part of the lowestlying conjugate pole $q= \pm \alpha_{1}+i \alpha_{0}$ (only the pole with $\alpha_{1}>0$ is shown) of a fluid of hard ellipsoids for varying densities in the isotropic fluid phase and varying aspect ratios $a / b=1(\square), 1.5(\square)$, $3.2(\times), 6(\nabla), 11(+), 25(\bigcirc)$ using the low-density approximation (22) with $l_{\text {max }}=10$ and employing the Parsons-Lee [32,33] Ansätze.

the $c_{l_{1} l_{2} l}(r)$ for hard ellipsoids obtained from the Parsons-Lee and Pynn-Wulff approximations, no pure imaginary pole was found. Consequently, the asymptotic decay is predicted to be exponentially damped oscillatory for all elongations. In this paper, we have limited ourselves to the asymptotic decay of pair correlations in bulk fluids of particles interacting with anisotropic pair potentials. It is interesting to investigate whether the pole structure given by $h_{l_{1} l_{2} l}(q)$ determines not only the decay of the total correlation functions in a bulk fluid but also the decay of the one body density profiles at wall-fluid interfaces. We plan to study this in future work.

\section{ACKNOWLEDGMENTS}

We thank René van Roij, Bob Evans, and Matthias Schmidt for stimulating discussions. We also thank René van Roij and Patrick Johnson for a critical reading of the manuscript. We acknowledge the Dutch National Computer Facilities Foundation for access to the SGI Origin 3800.

\section{APPENDIX A: BINARY FLUID MIXTURES}

The pole analysis can easily be extended to binary mixtures. The OZ equation for a binary $(\alpha, \beta)$ mixture in Fourier space reads [12]

$$
h_{\alpha \beta}(q)=c_{\alpha \beta}(q)+\sum_{\gamma} \rho_{\gamma} c_{\alpha \gamma}(q) h_{\gamma \beta}(q) \text {, }
$$

where $\rho_{\gamma}$ is the number density of species $\gamma$. It is convenient to write the OZ equation in a simple matrix equation of the form $A_{i j} h_{j}=c_{i}$, where component $j$ of vector $h$ is short for $j$ $=(\alpha \beta)$, the $q$ dependence is dropped for clarity, and the elements of matrix $A_{i j}$ are functions of $c_{\alpha \beta}$ : 


$$
\begin{aligned}
& \left(\begin{array}{cccc}
1-\rho_{1} c_{11} & -\rho_{2} c_{12} & & \\
-\rho_{1} c_{12} & 1-\rho_{2} c_{22} & & \\
& & 1-\rho_{1} c_{11} & -\rho_{2} c_{12} \\
& & -\rho_{1} c_{12} & 1-\rho_{2} c_{22}
\end{array}\right)\left(\begin{array}{l}
h_{11} \\
h_{21} \\
h_{12} \\
h_{22}
\end{array}\right) \\
& =\left(\begin{array}{l}
c_{11} \\
c_{21} \\
c_{12} \\
c_{22}
\end{array}\right)
\end{aligned}
$$

The coefficients matrix $A_{i j}$ has a block structure with identical blocks for each $\beta$. We define $\left|A^{(\beta)}\right|$ as the determinant of block $\beta$, which are identical for each $\beta$. It is straightforward to derive that $h_{j}=\left(A^{-1}\right)_{j i} c_{i}$ where $\left(A^{-1}\right)_{j i}=|A|_{i j} /|A|$ with $|A|$ the product of two identical determinants, $|A|=\Pi_{\beta}\left|A^{(\beta)}\right|$, and $|A|_{i j}=\left|A^{(\beta)}\right|_{i j} \Pi_{\alpha \neq \beta}\left|A^{(\alpha)}\right|$ the cofactor of matrixelement $A_{j i}$. Hence $\left(A^{-1}\right)_{j i}$ reduces to $\left(A^{-1}\right)_{j i}=\left|A^{(\beta)}\right|_{i j} /\left|A^{(\beta)}\right|$. The asymptotic behavior of the total correlation functions are determined by the poles and residues of $h_{j}$. As these are determined by the zeros of a denominator $\left|A^{(\beta)}\right|$, which is identical for each $\beta$, all $h_{j} \equiv h_{\alpha \beta}(q)$ exhibit the same pole structure and all $h_{\alpha \beta}(r)$ 's have the same asymptotic decay. However, the amplitudes are dependant on indices $\alpha \beta$. It is straightforward to extend the pole analysis to multicomponent mixtures yielding that there is only one decay length and (if appli- cable) one oscillatory wavelength that specifies the asymptotic decay of all partial total correlation functions $h_{\alpha \beta}(r)$.

\section{APPENDIX B: MOLECULAR FLUIDS}

In a similar way, the $\mathrm{OZ}$ equation for molecular fluids (11) can be expressed as a simple matrix equation of the form $A_{i j} h_{j}=c_{i}$. The component $j$ of vector $h$ corresponds to $j=\left(l_{1} l_{2} l\right)$. We give explicit expressions for $A_{i j}$ for $l_{\max }=2$. We first set $K_{l^{\prime \prime} l^{\prime} l_{3}}^{l_{1} l_{2} l}$ to one for all values of $\left(l^{\prime \prime}, l^{\prime}, l_{3}, l_{1}, l_{2}, l\right)$ and ignore the selection rules for the indices $l_{1} l_{2} l$. The matrix equation reads

$$
\left(\begin{array}{l|lll}
A_{i j}^{\left(l_{2}=0\right)} & \emptyset & \\
\hline & \emptyset & A_{i j}^{\left(l_{2}=2\right)}
\end{array}\right)\left(\begin{array}{l}
h_{000} \\
h_{002} \\
h_{200} \\
h_{202} \\
h_{020} \\
h_{022} \\
h_{220} \\
h_{222}
\end{array}\right)=\left(\begin{array}{c}
c_{000} \\
c_{002} \\
c_{200} \\
c_{202} \\
c_{020} \\
c_{022} \\
c_{220} \\
c_{222}
\end{array}\right),
$$

where $A_{i j}^{\left(l_{2}=0\right)}=A_{i j}^{\left(l_{2}=2\right)}$ and reads

$$
A_{i j}^{\left(l_{2}\right)}=\left(\begin{array}{cccc}
1-\hat{\rho} c_{000}-\hat{\rho} c_{002} & -\hat{\rho} c_{000}-\hat{\rho} c_{002} & -\hat{\rho} c_{020}-\hat{\rho} c_{022} & -\hat{\rho} c_{020}-\hat{\rho} c_{022} \\
-\hat{\rho} c_{000}-\hat{\rho} c_{002} & 1-\hat{\rho} c_{000}-\hat{\rho} c_{002} & -\hat{\rho} c_{020}-\hat{\rho} c_{022} & -\hat{\rho} c_{020}-\hat{\rho} c_{022} \\
-\hat{\rho} c_{200}-\hat{\rho} c_{202} & -\hat{\rho} c_{200}-\hat{\rho} c_{202} & 1-\hat{\rho} c_{220}-\hat{\rho} c_{222} & -\hat{\rho} c_{220}-\hat{\rho} c_{222} \\
-\hat{\rho} c_{200}-\hat{\rho} c_{202} & -\hat{\rho} c_{200}-\hat{\rho} c_{202} & -\hat{\rho} c_{220}-\hat{\rho} c_{222} & 1-\hat{\rho} c_{220}-\hat{\rho} c_{222}
\end{array}\right)
$$

We define $\hat{\rho}=(4 \pi)^{-3 / 2} \rho$. The coefficients matrix $A_{i j}$ decouples into separate subsets, which are all equal, for each value of $l_{2}$. However, if we include $K_{l^{\prime \prime} l^{\prime} l_{3}}^{l_{1} l_{2} l}$ and employ the selection rules, certain values of $l_{1}, l_{2}, l$ for the expansion coefficients vanish and, we find

$$
\left(\begin{array}{c|c}
A_{i j}^{\left(l_{2}=0\right)} & \emptyset \\
\hline \emptyset & A_{i j}^{\left(l_{2}=2\right)}
\end{array}\right)\left(\begin{array}{l}
h_{000} \\
h_{202} \\
h_{022} \\
h_{220} \\
h_{222}
\end{array}\right)=\left(\begin{array}{c}
c_{000} \\
c_{202} \\
c_{022} \\
c_{220} \\
c_{222}
\end{array}\right),
$$

where

$$
A_{i j}^{\left(l_{2}=0\right)}=\left(\begin{array}{cc}
1-\hat{\rho} c_{000} & -5 \hat{\rho} c_{022} \\
-\hat{\rho} c_{202} & 1-\sqrt{\frac{1}{5}}_{\hat{\rho} c_{220}}+\sqrt{\frac{10}{7}} \hat{\rho} c_{222}
\end{array}\right),
$$

and 


$$
A_{i j}^{\left(l_{2}=2\right)}=\left(\begin{array}{ccc}
1-\hat{\rho} c_{000} & -\sqrt{\frac{1}{5}} \hat{\rho} c_{022} & \sqrt{\frac{10}{7}} \hat{\rho} c_{022} \\
-\sqrt{5} \hat{\rho} c_{202} & 1-\sqrt{\frac{1}{5}} \hat{\rho} c_{220} & -\sqrt{5} \hat{\rho} c_{222} \\
-\sqrt{\frac{10}{7}} \hat{\rho} c_{202} & -\sqrt{\frac{1}{5}} \hat{\rho} c_{222} & 1-\sqrt{\frac{1}{5}} \hat{\rho} c_{220}-\frac{3}{7} \sqrt{\frac{5}{14}} \hat{\rho} c_{222}
\end{array}\right) .
$$

The coefficients matrix $A_{i j}$ has still a block structure and decouples into separate subsets corresponding to different values of $l_{2}$. However, the dimension of the subsets differ for different values of $l_{2}$. We define $\left|A^{\left(l_{2}\right)}\right|$ as the determinant of block $l_{2}$. The expansion coefficient can now be expressed as $h_{j}=\left(A^{-1}\right)_{j i} c_{i}$ where $\left(A^{-1}\right)_{j i}=|A|_{i j}|| A \mid$ with $|A|=\Pi_{l_{2}^{\prime}} \mid A^{\left(l_{2}{ }^{\prime}\right)}$, and $|A|_{i j}=\left|A^{\left(l_{2}\right)}\right|_{i j} \Pi_{l_{2}^{\prime} \neq l_{2}}\left|A^{\left(l_{2}^{\prime}\right)}\right|$. Hence, $\left(A^{-1}\right)_{j i}$ reduces to $\left(A^{-1}\right)_{j i}$ $=\left.\left|A^{\left(l_{2}\right)}{ }_{i j} /\right| A^{\left(l_{2}\right)}\right|^{2}$. The asymptotic behavior of the total correlation functions is determined by the poles and residues of $h_{j}$ $\equiv h_{l_{1} l_{2} l}(q)$ and are thus determined by the zeros of a denominator $\mid A^{\left(l_{2}\right)}$. Consequently, only the harmonics belonging to a particular subset corresponding to a certain value of $l_{2}$ share the same determinant and the same pole structure. Employing the symmetry property that the harmonic expansion coefficients are invariant under $l_{1} \leftrightarrow l_{2}$ permutation, we find that the determinants of different blocks, which are not equal in general, should share the zeros, i.e., $h_{l_{1} l_{2} l}(q)$ should exhibit the same pole structure as $h_{l_{2} l_{1} l}(q)$, resulting in the same asymptotic decay of $h_{l_{1} l_{2} l}(r)$ and $h_{l_{2} l_{1}}(r)$. More explicitly, in the case of $l_{\max }=2$, we find a common asymptotic decay for $h_{000}(r)$ and $h_{202}(r)$ and for $h_{022}(r), h_{220}(r)$, and $h_{222}(r)$. The $l_{1} \leftrightarrow l_{2}$ symmetry property yields that the asymptotic decay of $h_{202}(r)$ and $h_{022}(r)$ are equal and hence all $h_{l_{1} l_{2} l}(r)$ have the same asymptotic decay.
[1] J. G. Kirkwood, J. Chem. Phys. 7, 919 (1939).

[2] J. R. Henderson, Phys. Rev. E 50, 4836 (1994).

[3] R. Evans, J. R. Henderson, D. C. Hoyle, A. O. Parry, and Z. A. Sabeur, Mol. Phys. 80, 755 (1993).

[4] A. A. Chernov and L. V. Mikheev, Physica A 157, 1042 (1989).

[5] P. Tarazona, E. Chacon, M. Reinaldo-Falagan, and E. Velasco, J. Chem. Phys. 117, 3941 (2002).

[6] M. E. Fisher and B. Widom, J. Chem. Phys. 50, 3756 (1969).

[7] R. J. F. Leote de Carvalho, R. Evans, D. C. Hoyle, and J. R. Henderson, J. Phys.: Condens. Matter 6, 9275 (1994).

[8] C. Vega, R. F. Rull, and S. Lago, J. Chem. Phys. 51, 3146 (1995).

[9] R. J. F. Leote de Carvalho and R. Evans, Mol. Phys. 83, 619 (1994).

[10] R. J. F. Leote de Carvalho, R. Evans, and Y. Rosenfeld, Phys. Rev. E 59, 1435 (1999).

[11] W. E. Brown, R. J. F. Leote de Carvalho, and R. Evans, Mol. Phys. 88, 579 (1996).

[12] R. Evans, R. J. F. Leote de Carvalho, J. R. Henderson, and D. C. Hoyle, J. Chem. Phys. 100, 591 (1994).

[13] R. J. F. Leote de Carvalho and R. Evans, Mol. Phys. 92, 211 (1997).

[14] A. J. Archer and R. Evans, Phys. Rev. E 64, 041501 (2001).

[15] M. Schmidt, H. Löwen, J. N. Brader, and R. Evans, J. Phys.: Condens. Matter 14, 9353 (2002).

[16] A. J. Archer, C. N. Likos, and R. Evans, J. Phys.: Condens. Matter 14, 12031 (2002).
[17] C. Grodon, M. Dijkstra, R. Evans, and R. Roth, J. Chem. Phys. 121, 7869 (2004).

[18] C. Grodon, M. Dijkstra, R. Evans, and R. Roth, Mol. Phys. (to be published).

[19] M. Dijkstra and R. Evans, J. Chem. Phys. 112, 1449 (2000).

[20] R. D. Groot, J. P. van der Eerden, and N. M. Faber, J. Chem. Phys. 87, 2263 (1987).

[21] C. G. Gray and K. E. Gubbins, Theory of Molecular Fluids, Vol. 1 (Oxford University Press, New York, 1984).

[22] L. Blum and A. J. Torruella, J. Chem. Phys. 56, 303 (1972).

[23] I. M. Gelfand, M. M. Kapranov, and A. V. Zelevinsky, Discriminants, Resultants and Multidimensional Determinants (Birkhauser, Boston 1994).

[24] Handbook of Mathematical Functions with Formulas, Graphs, and Mathematical Tables, edited by M. Abramowitz and I. A. Stegun (Dover, New York, 1972).

[25] J. M. Haile and C. G. Gray, Chem. Phys. Lett. 76, 583 (1980).

[26] P. Bolhuis and D. Frenkel, J. Chem. Phys. 106, 666 (1997).

[27] M. P. Allen, C. P. Mason, E. de Miguel, and J. Stelzer, Phys. Rev. E 52, R25 (1995).

[28] N. H. Phuong and F. Schmid, J. Chem. Phys. 119, 1214 (2003).

[29] R. Pynn, J. Chem. Phys. 60, 4579 (1974).

[30] A. Wulf, J. Chem. Phys. 67, 2254 (1977).

[31] B. J. Berne and P. Pechukas, J. Chem. Phys. 64, 4213 (1972).

[32] J. D. Parsons, Phys. Rev. A 19, 1225 (1979).

[33] S. D. Lee, J. Chem. Phys. 87, 4972 (1987). 